\title{
No benefits of cryoablation over radiofrequency ablation in atrioventricular nodal re-entrant tachycardia
}

The largest study comparing the short-term and long-term outcomes of radiofrequency catheter ablation with cryoablation in patients with atrioventricular (AV) nodal re-entrant tachycardia (AVNRT) has been published. Radiofrequency is currently the standard treatment for this group of patients, but $1-2 \%$ of patients experience AV block, which necessitates pacemaker implantation. A pilot study showing that the two methods produced similar results led to the work published in Circulation. "In this trial, we used a larger tip catheter $(6 \mathrm{~mm})$

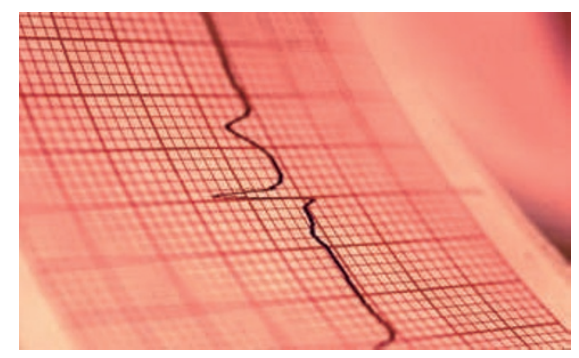

and applied an extra freeze at the successful site assuming that these features might improve the longevity and size of the lesion," says lead investigator Isabel Deisenhofer.

Patients with confirmed AVNRT were randomly assigned to radiofrequency ablation or cryoablation, and 496 patients were included in the final analysis at 6 months. The combined primary end point of short-term efficacy of the procedure, efficacy after 6 months, and ablation-induced AV block requiring pacemaker implantation occurred in $12.6 \%$ of the cryoablation group and $6.3 \%$ of the radiofrequency group $(P=0.018)$. No AV block occurred in the cryoablation group, and only one patient developed permanent complete AV block with radiofrequency. "The low frequency of AV block in the radiofrequency group most probably reflects the experience of the participating centers," comments Mats Jensen-Urstad from the Karolinska Institute, Sweden, who was not involved in the study. Any advantages of cryoablation were, however, limited by the significantly increased rates of AVNRT recurrence in this group (9.4\% versus $4.4 \% ; P=0.029)$. Creating larger lesions might prevent recurrence and needs to be investigated. Device failure was also higher and procedure duration longer in the cryoablation group than in the radiofrequency group.

"The use of cryoablation is limited by the high recurrence rate and seems to offer only negligible safety advantages over radiofrequency," says Deisenhofer. "We will use cryoablation only in cases with a very high risk of ablation-induced AV block."

Helene Myrvang

Original article Deisenhofer, I. et al. Cryoablation versus radiofrequency energy for the ablation of atrioventricular nodal reentrant tachycardia (the CYRANO Study): results from a large multicenter prospective randomized trial. Circulation 122, 2239-2245 (2010) 